PROCEEDINGS OF THE

AMERICAN MATHEMATICAL SOCIETY

Volume 128, Number 3, Pages 819-825

S 0002-9939(99)05073-X

Article electronically published on June 17, 1999

\title{
DISCRETE SPECTRUM OF ELECTROMAGNETIC DIRAC OPERATORS
}

\author{
NAOHIRO SUZUKI
}

(Communicated by David R. Larson)

\begin{abstract}
We consider the Dirac operators with electromagnetic fields on 2-dimensional Euclidean space. We offer the sufficient conditions for electromagnetic fields that the associated Dirac operator has only discrete spectrum.
\end{abstract}

\section{INTRODUCTION}

In this paper we consider electromagnetic Dirac operators on the 2-dimensional Euclidean space $\mathbf{R}^{2}$. Let $\left(L^{2}\left(\mathbf{R}^{2}\right)\right)^{2}$ be a Hilbert space

$$
\left(L^{2}(\mathbf{R})\right)^{2}=L^{2}\left(\mathbf{R}^{2}\right) \oplus L^{2}\left(\mathbf{R}^{2}\right)
$$

with inner product $(f, g) \equiv \int_{\mathbf{R}^{2}}\left(f_{1} \overline{g_{1}}+f_{2} \overline{g_{2}}\right) d x$ for $f=\left(f_{1}, f_{2}\right), g=\left(g_{1}, g_{2}\right) \in$ $\left(L^{2}\left(\mathbf{R}^{2}\right)\right)^{2}$. In this Hilbert space we introduce a Dirac operator with electromagnetic field $\mathbf{a}=\left(a_{j}(x)\right)_{j=1}^{2}$ and potential $V(x)$ by

$$
\mathbf{H}_{V}(\mathbf{a})=\sum_{j=1}^{2} \sigma_{j}\left(-i \frac{\partial}{\partial x_{j}}-a_{j}\right)+V,
$$

where $\left(\sigma_{j}\right)_{j=1}^{2}$ is a system of $2 \times 2$ Hermitian constant matrices with the anticommutation relations $\sigma_{j} \sigma_{k}+\sigma_{k} \sigma_{j}=2 \delta_{j, k} I$. Here $a_{j}$ and $V$ should be understood as operators of multiplication by real-valued functions $a_{j}(x)$ and $V(x)$, respectively. Let us assume $a_{j}(x), V(x) \in L_{l o c}^{\infty}\left(\mathbf{R}^{2}\right)$. Then it is well-known that $\mathbf{H}_{V}(\mathbf{a})$ is selfadjoint (cf. Chernoff [1]). In case $\mathbf{a}=\left(a_{j}(x)\right)_{j=1}^{2} \in\left(C^{1}\left(\mathbf{R}^{2}\right)\right)^{2}$, we define $B$ as the $2 \times 2$ skew-symmetric matrix whose $(j, k)$ component is $\partial a_{k} / \partial x_{j}-\partial a_{j} / \partial x_{k}$. We describe $b$ as $\partial a_{2} / \partial x_{1}-\partial a_{1} / \partial x_{2}$. By using this, we have

$$
B(x)=\left(\begin{array}{cc}
0 & b(x) \\
-b(x) & 0
\end{array}\right) .
$$

The norm of $B$ is given by $|B| \equiv|b|$. First we offer the conditions of electromagnetic fields that the associated Dirac operator has only discrete spectrum. For this end, we introduce a non-negative form on $\left(L^{2}\left(\mathbf{R}^{2}\right)\right)^{2}$ by

$$
h_{\mathbf{a}, V}(\phi, \psi)=\left(\mathbf{H}_{V}(\mathbf{a}) \phi, \mathbf{H}_{V}(\mathbf{a}) \psi\right), \quad \phi, \psi \in\left(C_{0}^{\infty}\left(\mathbf{R}^{2}\right)\right)^{2}
$$

Received by the editors September 12, 1997 and, in revised form, April 29, 1998. 1991 Mathematics Subject Classification. Primary 35P25; Secondary 81Q10.

Key words and phrases. Dirac operator, discrete spectrum, electromagnetic fields.

(C)1999 American Mathematical Society 
and define, for open sets $\Omega$ in $\mathbf{R}^{2}$

$$
e_{\mathbf{a}, V}(\Omega)=\inf \left\{h_{\mathbf{a}, V}(\phi, \phi) ;\|\phi\|=1, \operatorname{supp} \phi \subset \Omega, \phi \in\left(C_{0}^{\infty}\left(\mathbf{R}^{2}\right)\right)^{2}\right\} .
$$

Let us state our main theorem.

Main Theorem. The following four conditions are equivalent.

(a) $\mathbf{H}_{V}(\mathbf{a})$ has only discrete spectrum.

(b) $e_{\mathbf{a}, V}\left(W_{R}\right) \rightarrow \infty$ as $R \rightarrow \infty$, where $W_{R}=\{x|| x \mid>R\}$.

(c) $e_{\mathbf{a}, V}\left(Q_{x}\right) \rightarrow \infty$ as $|x| \rightarrow \infty$, where $Q_{x}=\{y|| x-y \mid<1\}$.

(d) There exists a real-valued continuous function $\lambda(x)$ on $\mathbf{R}^{2}$ such that $\lambda(x) \rightarrow$ $\infty$ as $|x| \rightarrow \infty$ and that

$$
h_{\mathbf{a}, V}(\phi, \phi) \geqq \int_{\mathbf{R}^{2}} \lambda(x)|\phi(x)|^{2} d x
$$

for all $\phi(x) \in\left(C_{0}^{\infty}\left(\mathbf{R}^{2}\right)\right)^{2}$.

As an application, we give a sufficient condition that $\mathbf{H}_{V}(\mathbf{a})$ has only discrete spectrum.

Theorem 1.1. On the 2-dimensional Euclidean space, we assume that $\mathbf{a}=$ $\left(a_{j}(x)\right)_{j=1}^{2} \in\left(C^{2}\left(\mathbf{R}^{2}\right)\right)^{2}$ and $V(x) \in C^{1}\left(\mathbf{R}^{2}\right)$ and that the following three conditions (1.6), (1.7) and (1.8) are fulfilled. Then $\mathbf{H}_{V}(\mathbf{a})$ has only discrete spectrum.

$$
\begin{aligned}
& \lim _{|x| \rightarrow \infty} \frac{|b|}{V^{2}}>2, \\
& \lim _{|x| \rightarrow \infty}|V|=\infty, \\
& \lim _{|x| \rightarrow \infty} \frac{\nabla V}{V^{2}}=0 .
\end{aligned}
$$

Iwatsuka got a similar result to the Main Theorem in the case where $\mathbf{H}_{V}(\mathbf{a})$ is a Schrödinger operator. However, the conditions for Schrödinger operators have only discrete spectrum are quite different from those for Dirac operators. In fact, under the conditions $V=0$ and $\lim _{|x| \rightarrow \infty}|b|=\infty$, Schrödinger operators have only discrete spectrum, but Dirac operators may have an essential one. We know a similar fact as above when $b=0$ and $\lim _{|x| \rightarrow \infty} V=\infty$ (cf. Thaller [6]).

\section{Proof of the Main Theorem}

Let $E$ be the spectral measure associated with $\mathbf{H}_{V}(\mathbf{a}) . \sigma_{\mathrm{ess}}\left(\mathbf{H}_{V}(\mathbf{a})\right)$ is defined by

$$
\sigma_{\text {ess }}\left(\mathbf{H}_{V}(\mathbf{a})\right)=\{\mu \in \mathbf{R} \mid \operatorname{dim} \operatorname{Ran}(E(\mu-\varepsilon, \mu+\varepsilon))=\infty \text { for all } \varepsilon>0\},
$$

where $\operatorname{Ran}(\cdot)$ denotes the range of an operator. Note that

$$
\sigma_{\text {ess }}\left(\mathbf{H}_{V}(\mathbf{a})\right)=\phi \quad \text { if and only if } \quad \mathbf{H}_{V}(\mathbf{a}) \text { has only discrete spectrum. }
$$

We need the following lemma to prove the Main Theorem.

Lemma 2.1. Let $s>0$. Then the following conditions are equivalent.

(a) $\inf \left\{|\lambda| \mid \lambda \in \sigma_{\text {ess }}\left(\mathbf{H}_{V}(\mathbf{a})\right)\right\} \geqq s$.

(b) $\lim _{R \rightarrow \infty} e_{\mathbf{a}, V}\left(W_{R}\right) \geqq s^{2}$, where $W_{R}=\{x|| x \mid>R\}$. 
(c) There exists a real-valued continuous function $\lambda(x)$ on $\mathbf{R}^{2}$ such that

$$
\liminf _{|x| \rightarrow \infty} \lambda(x) \geqq s^{2} \quad \text { and } \quad h_{\mathbf{a}, V}(\phi, \phi) \geqq \int_{\mathbf{R}^{d}} \lambda(x)|\phi(x)|^{2} d x
$$

for all $\phi(x) \in\left(C_{0}^{\infty}\left(\mathbf{R}^{2}\right)\right)^{2}$.

Proof. (a) $\Rightarrow\left(\right.$ b): Suppose that (b) does not hold. There exists some $s^{\prime}>0$ such that $\lim _{R \rightarrow \infty} e_{\mathbf{a}, V}\left(W_{R}\right)<{s^{\prime}}^{2}<s^{2}$. Since $e_{\mathbf{a}, V}\left(W_{R}\right)$ is increasing in $R$ by (1.5), we notice that $e_{\mathbf{a}, V}\left(W_{R}\right)<s^{\prime 2}$ for all $R>0$. Hence, one can choose successively a sequence $\left\{\phi_{k}\right\}_{k=1}^{\infty} \subset\left(C_{0}^{\infty}\left(\mathbf{R}^{2}\right)\right)^{2}$ such that

$$
\left\{\begin{array}{l}
\left\|\phi_{k}\right\|=1, \\
h_{\mathbf{a}, V}\left(\phi_{k}, \phi_{k}\right)<s^{\prime 2}, \\
\operatorname{supp} \phi_{k} \subset\left\{x\left|a_{k}<\right| x \mid<a_{k+1}\right\},
\end{array}\right.
$$

for some increasing sequence $\left\{a_{k}\right\}_{k=1}^{\infty}$ such that $a_{k} \rightarrow \infty$ as $k \rightarrow \infty$. Let $E(t)=$ $E((\infty, t])$. If $s^{\prime}<t<s$, (a) implies that $E(t)-E(-t)$ is compact; hence

$$
\lim _{k \rightarrow \infty}\left\|E(t) \phi_{k}\right\|^{2}=\lim _{k \rightarrow \infty}\left\|E(-t) \phi_{k}\right\|^{2} .
$$

Therefore we have

$$
\begin{aligned}
& h_{\mathbf{a} V}\left(\phi_{k}, \phi_{k}\right)=\int_{(-\infty, \infty)} \mu^{2} d\left\|E(\mu) \phi_{k}\right\|^{2} d x \\
& \geqq \int_{(t, \infty)} t^{2} d\left\|E(\mu) \phi_{k}\right\|^{2} d x+\int_{(-\infty,-t)} t^{2} d\left\|E(\mu) \phi_{k}\right\|^{2} d x \\
& =t^{2}\left(\left\|\phi_{k}\right\|^{2}-\left\|E(t) \phi_{k}\right\|^{2}+\left\|E(-t) \phi_{k}\right\|^{2}\right) \rightarrow t^{2} \text { as } k \rightarrow \infty .
\end{aligned}
$$

But this contradicts $(2.2)$.

(b) $\Rightarrow$ (c): Let $\left\{\xi_{k}\right\}_{k=0,1,2, \ldots}$ be a sequence of real-valued functions of class $C_{0}^{\infty}\left(\mathbf{R}^{2}\right)$ such that

$$
\left\{\begin{aligned}
\sum_{k=0}^{\infty} \xi_{k}^{2}(x) & =1, \\
\xi_{k}(x) & =\xi_{1}\left(\frac{x}{2^{k-1}}\right) \quad(k \geqq 1), \\
\operatorname{supp} \xi_{0} & \subset\{x|| x \mid<2\}, \\
\operatorname{supp} \xi_{k} & \subset\left\{x\left|2^{k-1}<\right| x \mid<2^{k+1}\right\} \quad(k \geqq 1) .
\end{aligned}\right.
$$

See Iwatsuka [3] for the existence of such a sequence. By direct computation,

$$
\left\{\begin{array}{c}
\mathbf{H}_{V}(\mathbf{a})\left(\xi^{2} \phi\right)=\xi^{2} \mathbf{H}_{V}(\mathbf{a}) \phi-\sum_{j=1}^{2} 2 i \sigma_{j} \xi \frac{\partial \xi}{\partial x_{j}} \phi, \\
\mathbf{H}_{V}(\mathbf{a})(\xi \phi)=\xi \mathbf{H}_{V}(\mathbf{a}) \phi-\sum_{j=1}^{2} i \sigma_{j} \frac{\partial \xi}{\partial x_{j}} \phi
\end{array}\right.
$$


for $\phi \in\left(C_{0}^{\infty}\left(\mathbf{R}^{2}\right)\right)^{2}$, and a real-valued function $\xi \in C^{\infty}\left(\mathbf{R}^{2}\right)$. By simple calculation, we have

$$
\begin{aligned}
& h_{\mathbf{a}, V}\left(\phi, \xi_{k}^{2} \phi\right)-h_{\mathbf{a}, V}\left(\xi_{k} \phi, \xi_{k} \phi\right)=\left(\sum_{j=1}^{2} i \sigma_{j} \frac{\partial \xi_{k}}{\partial x_{j}} \phi, i \sigma_{j} \frac{\partial \xi_{k}}{\partial x_{j}} \phi\right) \\
= & \left(\phi,\left(-\sum_{j=1}^{2} i \sigma_{j} \frac{\partial \xi_{k}}{\partial x_{j}}\right)\left(\sum_{j=1}^{2} i \sigma_{j} \frac{\partial \xi_{k}}{\partial x_{j}}\right) \phi\right)=\left\|\left|\nabla \xi_{k}\right| \phi\right\|^{2} .
\end{aligned}
$$

We obtain

$$
\begin{aligned}
h_{\mathbf{a}, V}(\phi, \phi) & =\mathbf{R} e\left(\sum_{k=0}^{\infty} h_{\mathbf{a}, V}\left(\phi, \xi_{k}^{2} \phi\right)\right) \\
& =\sum_{k=0}^{\infty} h_{\mathbf{a}, V}\left(\xi_{k} \phi, \xi_{k} \phi\right)-\sum_{k=0}^{\infty}\left\|\left|\nabla \xi_{k}\right| \phi\right\|^{2},
\end{aligned}
$$

where $|\nabla \xi|=\sqrt{\sum_{j=1}^{2}\left(\partial \xi / \partial x_{j}\right)^{2}}$. Let $\lambda(x)=\sum_{k=0}^{\infty}\left(e_{k} \xi_{k}(x)^{2}-\left|\nabla \xi_{k}\right|^{2}\right)$, where $e_{0}=e_{\mathbf{a}, V}(\{x|| x \mid<2\})$ and $e_{k}=e_{\mathbf{a}, V}\left(W_{2^{k-1}}\right), k=1,2, \cdots$, now one notices that (c) holds.

(c) $\Rightarrow$ (a): Suppose that (a) does not hold. Choose $\sigma \in \sigma_{\text {ess }}\left(\mathbf{H}_{V}(\mathbf{a})\right)$ such that $|\sigma|<s$, and a sequence $\left\{u_{k}\right\}_{k=1}^{\infty} \in \mathbf{D}\left(\mathbf{H}_{V}(\mathbf{a})\right)$ such that

$$
\left\{\begin{array}{l}
\left\|u_{k}\right\|=1, \\
u_{k} \rightarrow 0 \text { weakly as } k \rightarrow \infty, \\
\left\|\mathbf{H}_{V}(\mathbf{a}) u_{k}-\sigma u_{k}\right\| \rightarrow 0 \text { as } k \rightarrow \infty .
\end{array}\right.
$$

From (c) for any $0<\varepsilon<s^{2}-\sigma^{2}$, there exists $M>0$ such that $\lambda(x)>s^{2}-\varepsilon$ for $x$ : $|x| \geqq M$. Then we have

$$
\begin{aligned}
h_{\mathbf{a}, V}\left(u_{k}, u_{k}\right) & \geqq \int_{|x|>M} \lambda(x)\left|u_{k}(x)\right|^{2} d x+\int_{|x| \leqq M} \lambda(x)\left|u_{k}(x)\right|^{2} d x \\
& \geqq\left(s^{2}-\varepsilon\right) \int_{|x|>M}\left|u_{k}(x)\right|^{2} d x-M_{\lambda} \int_{|x| \leqq M}\left|u_{k}(x)\right|^{2} d x,
\end{aligned}
$$

where $M_{\lambda}=\max _{|x| \leqq M}|\lambda(x)|$.

It suffices to find a subsequence $\left\{u_{k^{\prime}}\right\}$ of $\left\{u_{k}\right\}$ such that $\lim _{k^{\prime} \rightarrow \infty} \int_{|x| \leqq M}\left|u_{k^{\prime}}\right|^{2} d x=0$. In fact, it implies that $\sigma^{2}=\lim _{k^{\prime} \rightarrow \infty} h_{\mathbf{a} V}\left(u_{k^{\prime}}, u_{k^{\prime}}\right) \geqq s^{2}-\varepsilon$, which contradicts $\varepsilon<$ $s^{2}-\sigma^{2}$. We can choose the following real-valued functions of class $C^{\infty}\left(\mathbf{R}^{2}\right)$ :

$$
\begin{cases}\varphi_{0}^{2}(x)+\varphi_{1}^{2}(x)=1, & \\ \varphi_{0}(x)=1 & \text { if }|x| \leq M, \\ \varphi_{0}(x)=0 & \text { if }|x| \geq M+1 .\end{cases}
$$

In similar fashion to (2.4), we get

$$
h_{\mathbf{a}, V}\left(u_{k}, u_{k}\right)=\sum_{l=0}^{1} h_{\mathbf{a}, V}\left(\varphi_{l} u_{k}, \varphi_{l} u_{k}\right)-\sum_{l=0}^{1}\left\|\left|\nabla \varphi_{l}\right| u_{k}\right\|^{2} .
$$


Hence, we obtain

$$
h_{\mathbf{a}, V}\left(\varphi_{0} u_{k}, \varphi_{0} u_{k}\right) \leq h_{\mathbf{a}, V}\left(u_{k}, u_{k}\right)+\sum_{l=0}^{1}\left\|\left|\nabla \varphi_{l}\right| u_{k}\right\|^{2},
$$

which proves that $h_{\mathbf{a}, V}\left(\varphi_{0} u_{k}, \varphi_{0} u_{k}\right)$ is bounded. We define that $\Omega_{M+1}=$ $\{x|| x \mid \leq M+1\}$, which includes the support of $\varphi_{0}$. By the assumption that $\mathbf{a}_{j}, V \in L_{l o c}^{\infty}$, we notice that $\left\|\mathbf{H}_{V}(\mathbf{a})\left(\varphi_{0} \cdot\right)\right\|$ is equivalent to $\left\|\varphi_{0} \cdot\right\|_{H_{1}}$. Because the embedding map from $\left(H_{1}\left(\Omega_{M+1}\right)\right)^{2}$ to $\left(L^{2}\left(\Omega_{M+1}\right)\right)^{2}$ is compact, there exists a subsequence $\left\{u_{k^{\prime}}\right\}$ of $\left\{u_{k}\right\}$ such that $\lim _{k^{\prime} \rightarrow \infty} \int_{\mathbf{R}^{2}}\left(\varphi_{0}(x)\left|u_{k^{\prime}}\right|\right)^{2} d x=0$. We have

$$
0 \leq \lim _{k^{\prime} \rightarrow \infty} \int_{M}\left|u_{k^{\prime}}\right|^{2} d x \leq \lim _{k^{\prime} \rightarrow \infty} \int_{\mathbf{R}^{2}}\left|\varphi_{0}(x) u_{k^{\prime}}\right|^{2} d x=0,
$$

which completes the proof.

Owing to this lemma, the proof of the Main Theorem is analogous to that by Iwatsuka [3, Main Theorem].

\section{Sufficient COnditions For $\mathbf{H}_{V}(\mathbf{a})$ To HAVE Only Discrete SPECTRum}

Through this section, we assume that $a_{j}$ and $V$ are smooth, and we shall offer sufficient conditions for $\mathbf{H}_{V}(\mathbf{a})$ to have only discrete spectrum. We have by selfadjointness of $\mathbf{H}_{V}(\mathbf{a})$.

$$
h_{\mathbf{a}, V}(\phi, \phi)=\left(\mathbf{H}_{V}(\mathbf{a}) \phi, \mathbf{H}_{V}(\mathbf{a}) \phi\right)=\left(\mathbf{H}_{V}(\mathbf{a})^{2} \phi, \phi\right) .
$$

We have by direct calculation,

$$
\begin{aligned}
\mathbf{H}_{V}(\mathbf{a})^{2}= & -\sum_{j=1}^{2}\left(\frac{\partial}{\partial x_{j}}-i a_{j}\right)^{2}+V^{2}+i b \sigma_{1} \sigma_{2} \\
& +V \sum_{j=1}^{2} \sigma_{j}\left(-i \frac{\partial}{\partial x_{j}}-a_{j}\right)+\sum_{j=1}^{2} \sigma_{j}\left(-i \frac{\partial}{\partial x_{j}}-a_{j}\right) V \\
= & -\sum_{j=1}^{2}\left(\frac{\partial}{\partial x_{j}}-i a_{j}+i \sigma_{j} V\right)^{2}-V^{2}+i b \sigma_{1} \sigma_{2} .
\end{aligned}
$$

Hence, we obtain

$$
h_{\mathbf{a}, V}(\phi, \phi)=\sum_{j=1}^{2}\left\|\Pi_{j} \phi\right\|^{2}-\|V \phi\|^{2}+\left(i b \sigma_{1} \sigma_{2} \phi, \phi\right),
$$

where

$$
\Pi_{j}=\frac{\partial}{\partial x_{j}}-i a_{j}+i \sigma_{j} V
$$

We get by simple calculation,

$$
\Pi_{2} \Pi_{1}-\Pi_{1} \Pi_{2}=i b+2 \sigma_{1} \sigma_{2} V^{2}+i \sigma_{1} \frac{\partial V}{\partial x_{2}}-i \sigma_{2} \frac{\partial V}{\partial x_{1}},
$$

which plays an important role in this section. 
Proof of Theorem 1.1. There exists $N>0$ such that if $|x| \geq N$, then

$$
\begin{aligned}
& V \neq 0, \\
& |b|>2 V^{2}, \\
& \left|\frac{\nabla V}{V^{2}}\right|<\frac{1}{4} .
\end{aligned}
$$

One can choose a function $\phi \in\left(C_{0}^{\infty}\left(\mathbf{R}^{2}\right)\right)^{2}$. We assume that $\operatorname{supp} \phi \subset W_{R}$ and $R>N$. We have by (3.5), (3.6) and (3.8).

$$
\begin{aligned}
2\left\|\Pi_{1} \phi\right\|\left\|\Pi_{2} \phi\right\| & \geq\left|\left(\Pi_{2} \phi, \Pi_{1} \phi\right)-\left(\Pi_{1} \phi, \Pi_{2} \phi\right)\right| \\
& \geq\left|\left(\left(\Pi_{2} \Pi_{1}-\Pi_{1} \Pi_{2}\right) \phi, \phi\right)\right| \\
& \geq\left|\left(\left(i b+2 \sigma_{1} \sigma_{2} V^{2}\right) \phi, \phi\right)\right|-\frac{1}{2}\|V \phi\|^{2} .
\end{aligned}
$$

We can regard $\sigma_{1}, \sigma_{2}$ as

$$
\sigma_{1}=\left(\begin{array}{cc}
0 & 1 \\
1 & 0
\end{array}\right), \sigma_{2}=\left(\begin{array}{cc}
0 & -i \\
i & 0
\end{array}\right)
$$

Now we can choose such a coordinate on $\mathbf{R}^{2}$ that $b>0$ on $W_{R}$. Then we obtain by (3.7) and (3.9),

$$
\begin{aligned}
& \left\|\Pi_{1} \phi\right\|^{2}+\left\|\Pi_{2} \phi\right\|^{2}+\left(i b \sigma_{1} \sigma_{2} \phi, \phi\right) \\
& \geq\left|\int_{\mathbf{R}^{2}} i\left(\phi_{1}, \phi_{2}\right)\left(\begin{array}{cc}
b+2 V^{2} & 0 \\
0 & b-2 V^{2}
\end{array}\right)\left(\frac{\bar{\phi}_{1}}{\bar{\phi}_{2}}\right) d x\right| \\
& +\int_{\mathbf{R}^{2}}\left(\phi_{1}, \phi_{2}\right)\left(\begin{array}{cc}
-b & 0 \\
0 & b
\end{array}\right)\left(\begin{array}{l}
\bar{\phi}_{1} \\
\bar{\phi}_{2}
\end{array}\right) d x-\frac{1}{2}\|V \phi\|^{2} \\
& \geq \int_{\mathbf{R}^{2}}\left(\phi_{1}, \phi_{2}\right)\left(\begin{array}{cc}
b+2 V^{2} & 0 \\
0 & 0
\end{array}\right)\left(\begin{array}{l}
\bar{\phi}_{1} \\
\bar{\phi}_{2}
\end{array}\right) d x \\
& +\int_{\mathbf{R}^{2}}\left(\phi_{1}, \phi_{2}\right)\left(\begin{array}{cc}
-b & 0 \\
0 & 2 V^{2}
\end{array}\right)\left(\begin{array}{l}
\bar{\phi}_{1} \\
\bar{\phi}_{2}
\end{array}\right) d x-\frac{1}{2}\|V \phi\|^{2} \\
& \geq \frac{3}{2}\|V \phi\| .
\end{aligned}
$$

Hence, by (3.3), we have

$$
h_{\mathbf{a}, V}(\phi, \phi) \geq \frac{1}{2} \inf _{x \in W_{R}} V^{2}\|\phi\|^{2} .
$$

Therefore, $e_{\mathbf{a}, V}\left(W_{R}\right) \rightarrow \infty$ as $R \rightarrow \infty$, which completes the proof.

\section{ACKNOWLEDGEMENT}

The author wishes to express his gratitude to Professor H. Matsumoto and Assistant Professor M. Sugiura at Nagoya University for valuable suggestions and kind encouragements.

\section{REFERENCES}

1. P. R. Chernoff, Schrödinger and Dirac operators with singular potentials and hyperbolic equations, Pac. J. Math. 72 (1977), 361-382. MR 58:23150

2. Colin de Verdiere, L'asymptotique de Weyl pour les bouteilles magnétiques, Comm. Math. Phys. 105 (1986), 327-335. MR 87k:58273 
3. A. Iwatsuka, Magnetic Schrödinger operators with compact resolvent, J. Math. Kyoto Univ. 26-3 (1986), 357-374. MR 87j:35287

4. M. Reed and B. Simon, Methods of modern mathematical physics, II, Fourier Analysis, Selfadjointness, Academic Press, New York, 1975. MR 58:12429b

5. _ Methods of modern mathematical physics, IV, Analysis of Operators, Academic Press, New York, 1978. MR 58:12429c

6. B. Thaller, The Dirac equation, Springer-Verlag, Berlin/Heidelberg, 1992. MR 94k:81056

49-9 Shinsakae, Shinden, Anjo, Aichi, 446-0061 Japan

E-mail address: nhsuzuki@cb3.so-net.or.jp 